\title{
QoS Aware Routing Protocol to Improve Packet Transmission in Shadow-Fading Environment for Mobile Ad Hoc Networks
}

\author{
T. Sangeetha ${ }^{1,2}$, K. K. Venkatesh ${ }^{1,2}$, Rajesh ${ }^{1,2}$, M. S. K. Manikandan ${ }^{1,3}$ \\ ${ }^{1}$ Department of Electronics and Communication Engineering, Madurai, India \\ ${ }^{2}$ SACS MAVMM Engineering College, Madurai, India \\ ${ }^{3}$ Thiagarajar College of Engineering, Madurai, India \\ Email: sangee4contact@gmail.com, venkateshkk113@gmail.com, smartrajesh9@gmail.com,manimsk@tce.edu
}

Received July 2013

\begin{abstract}
In Shadow-Fading model, it is difficult to achieve higher Packet Delivery Ratio (PDR) due to the effects of large scale fading. The main goal of this paper is to improve the PDR in Shadow-Fading environment. To achieve this objective a protocol has been proposed that discovers backup routes for active sessions. These backup routes salvage the packets of active session in case of active route failure. It is found by research that proactively maintaining backup routes for active sessions can noticeably improve the PDR in Shadow-Fading environment. This protocol has been implemented with a mechanism of having backup routes and simulations have been conducted by using both node disjoint paths and link disjoint paths. Comparisons have been made between new protocol as well as AODV protocol. Simulation has been carried out using Network Simulator 2 (NS2) and the results show that the proposed QoSAR protocol exhibits higher PDR than AODV protocol in Shadow-fading environment.
\end{abstract}

Keywords: Shadow-Fading Model; Adhoc On Demand Distance Vector Routing (AODV); Backup Routes; Node Disjoint Paths; Link Disjoint Paths; Packet Delivery Ratio (PDR); Quality of Service Aware Routing (QoSAR)

\section{Introduction}

In Mobile Ad hoc Networks (MANETs), the provision of Quality of Service (QoS) guarantees is much more challenging than in wireline networks, mainly due to node mobility, multihop communications, contention for channel access, and a lack of central coordination. QoS guarantees are required by most multimedia and other time or error sensitive applications. The difficulties in the provision of such guarantees have limited the usefulness of MANETs. The QoS routing protocol is an integral part of any QoS solution since its function is to discover which nodes, if any, are able to serve applications' requirements. Consequently, it also plays a crucial role in data session admission control.

MANET applications are nowadays used in military and in emergencies, entertainments and outdoor industry environments where centralized administration is difficult and costly to install [1]. Two nodes can communicate if they are within each other's radio range, otherwise, routers if they are out of range, thereby it becomes multipath routing. These networks have several salient fea- tures like rapid exploitation, robustness, flexibility, intrinsic mobility support, highly dynamic network topology, the limited battery power of mobile devices, limited capacity and asymmetric or unidirectional links [2].

Routing has always been one of the key challenges in Mobile Adhoc Networks and the challenge become more difficult when the network size increases [5]. Many multi path routing protocols have been proposed for Ad Hoc Networks [3]. The routing protocols mainly are either table-driven (Proactive) or on-demand (reactive) routing protocols. Many hybrid routing protocols having the combination of functionality of both proactive as well as reactive routing protocol are also proposed. The proactive routing protocols periodically update the routing tables. When there is a request to forward message the routes are available in the routing table. On the contrary reactive routing search the route when there is a request for it. In the route search operation the reactive routing protocols, find multiple paths for the same source and destination pair. One out of these multiple paths is selected to forward messages to the destination.

The basic idea of On-Demand routing protocol is to 
search route when it is required. Due to the mobility characteristic of MANET nodes and Dynamic Topology of the network, there are always multiple paths available between the sources to destination pair. The On Demand routing mechanism suggests using the route that is considered best according to the required Quality of Service for the transmission, as in [3] hop count, is the parameter for selecting a route. Once the best route out of searched route is chosen, the other routes are not given any considerations. But maintaining information about these additional routes will be more advantageous [2-4]. These additional routes can be used as backup routes in the events of link failures. In our paper the route failure can be overcome by disjointness for shadow fading environments.

This paper mainly focuses on the idea of finding multiple paths (Multi-path) using Node Disjoint and Link Disjoint paths to improve Packet Delivery Ratio (PDR) of the network in Shadow fading environments. In addition to this, the study also targets to evaluate the performances of AODV and QoS Aware Routing (QOSAR) protocols based on PDR and throughput. The organization of the rest of the paper is as follows. Section 2 QoS routing challenges in MANET. Section 3 briefs about the related work. Section 4 presents the discussion about proposed routing protocols and in Section 5 Result and Discussion.

\section{QoS Routing Challenges In MANET}

The design of efficient routing protocols is a critical issue for both wired and wireless networks. QoS-Aware Routing (QoSAR) protocols are facing the following design issues and constraints. These issues and constraints must be kept in mind while designing these protocols.

\subsection{Node Mobility}

A MANET consists of mobile nodes. Nodes form the network only when they are in the communication range of each other. If they move out of range, link between the two nodes is broken. At times, failure of a single link can lead to the major network partitioning. Hence, mobility of the nodes is a major challenging issue for a stable network. Also, failure of certain links results in routing decisions to be made again.

\subsection{Lack of Centralized Control}

The advantage of an ad-hoc network is that it is formed spontaneously without fixed architecture. Participant nodes or members can change their positions dynamically and can join or leave the network independently. Due to this nature of mobile ad-hoc networks it is difficult to provide centralized control. Thus it is difficult to achieve efficient and fair Medium Access Control (MAC). Thus those com- munication protocols are preferred which utilize only locally available states and operate in a completely distributed manner [6]. This increases complexity and an algorithm's overheads, as information about the participant nodes must be collected efficiently. There is no central entity to collect resource state information and admission decisions. Instead of central administration, nodes must make decisions based on available network resources, which may lead to potential inaccuracies. Due to lack of infrastructure the control and management of the network is distributed among the nodes.

\subsection{The Unreliable Wireless Channels}

Due to interference from the other transmissions, multi-path fading and shadowing effects the received signal are flat to bit errors. Such errors may lead to packets being not decidable. However, persistent packet error can result in link failure, leading to re-routing, increased packet delay and congestion and packet dropping [7].

\subsection{Multiple Paths}

To send data from a source to destination, a path has to be found beforehand. If a single path is established, sending all the traffic on it will deplete all the nodes faster. Also, in case of path failure, alternate path acts as a backup path. Thus, establishing multiple paths aids not only in traffic engineering but also prevents faster network degradation.

\subsection{Node-Disjoint Paths}

Multiple paths between the two nodes can be any link disjoint or node disjoint. Multiple link disjoint paths may have one node common among more than one path. Thus, traffic load on this node will be much higher than the other nodes of the paths. As a result, this node tends to die much earlier than the other nodes, primary to the paths to break down much earlier. Thus, the presence of node disjoint paths prolongs the network lifetime by reducing the energy fall rate of a particular node [8-9].

\section{Related Works}

This section briefs about the research work on the idea of multi-path routing in Mobile Ad hoc networks.

\subsection{Ad Hoc On-Demand Distance Vector Routing}

In this paper titled "Ad Hoc On-Demand Distance Vector Routing”, Feb- 1999, the authors C.E. Perkins and E.M. Royer have presented a distance vector algorithm that is suitable for use with ad-hoc networks. In AODV each Mobile Host operates as a specialized router, and routes are obtained as needed (i.e., on-demand) with little or no 
reliance on periodic advertisements. Their simulation shows that on-demand route establishment with AODV is quick i.e., and accurate. AODV is a reactive routing protocol, meaning that it provides a route to a destination only on demand. Because AODV does not require global periodic routing advertisements, the demand on the overall available bandwidth to the mobile nodes is substantially less than in those protocols that do necessitate such advertisements.

The main difference between AODV and DSR $[5,11]$ stems out from the fact that DSR uses source routing in which data packet carries the complete path to be traversed. In AODV, the source node and the intermediate packet nodes store the next hop information corresponding to each flow for data packet transmission. In this protocol, the source node floods the Route Request packet in the network when a route is not available for the desired destination. When an intermediate node receives a request for route, it either forwards the request or prepares a reply for that route if it has a valid route to the destination. The validity of a route at the intermediate node is determined by comparing the sequence number at the intermediate node with the destination sequence number in the Route Request packet. It may thus obtain multiple routes to different destinations from a single Route Request. Multiple Route Reply packets in response to a single Route Request packet lead to heavy control overhead. AODV avoids the counting-to-infinity problem of other distance-vector protocols by using sequence numbers on route updates. AODV has the ability for both unicast and multicast routing. But considering this case the AODV protocol not perform well in shadow fading environments its proved in our simulations.

\subsection{QoS Routing Solutions}

In this paper titled "A Survey of QoS routing solutions for Mobile Ad Hoc Networks", Apr-June 2007 the authors L. Hanzo II and R. Tafazolli have presented a QoS routing solutions that is suitable for use with ad-hoc networks $[10,13]$.

This paper offers an up-to-date survey of most major contributions to the pool of QoS routing solutions for MANETs published. It includes a thorough overview of QoS routing metrics, bandwidth, factors affecting performance and classify the protocols found in the literature. The aim of MAC protocol was to provide a basic best-effort level of service to ensure network operation in the face of an unpredictable and shared wireless communication medium and to maintain a network topology view and routes in the face of failing links and mobile devices. QoS routing protocols play a major part in a QoS mechanism, since it is their task to find which nodes, if any, can serve an application's requirements. Therefore, the QoS routing protocol also plays a major part in Ses- sion Admission Control (SAC), since that is dependent on the discovery of a route that can support the requested QoS.

The majority of the solutions proposed in this literature till now have focused on providing QoS based on two metrics: throughput and delay. Of these, the more common is throughput. This is most likely because assured throughput is somewhat of a "lowest common denominator" requirement; most voice or video applications require some level of guaranteed throughput in addition to their other constraints. The proposed protocol will improve the QoS based on the above two metrics.

\section{Protocol Implementation}

In this section, we describe the implementation of proposed protocol, which exploits the knowledge of alternative or backup routes to a source's destination in order to improve the robustness of throughput-QoS assurances in the face of route failures. The main goal of this paper is to improve the Packet Delivery Ratio (PDR) in shadow fading environments. Thus by using the proposed protocol Quality of Service (QoS) can be improved as much as possible. Hence in the following sections we are going to use the name as QoSAR (Qos Aware Routing) for the proposed protocol.

In shadow fading environments, it is difficult to achieve high PDR only with the use of primary routes from source to destination. Idea behind QOSAR is to find multiple routes between source and destination. Hence it improves PDR as well as it gives solution to the route failures.

In the newly proposed QoSAR protocol, once the session is ready to transmit, a backup route for that session must be found. We have considered two mechanisms to find multiple routes as follows:

1) Link Disjoint Paths

2) Node Disjoint Paths

\subsection{Link Disjoint Paths}

Link disjoint paths are paths between sources to destination which have no overlapping links. The backup route is selected in such a way they are "sufficiently disjoint" i.e., it includes no more than half of the links in the current/primary route [12].

In Figure 1 three paths (shown in red color) between sources to destination are called as link disjoint paths. If the primary and backup routes are selected in such a way that, they are sufficiently link disjoint then the route failure is minimized.

If a new backup route must be discovered, the search packet, referred to as RReq backup carries a copy of the session's primary route. To avoid fully flooding the network with the RReq-backup, once the RReq backup has traveled at least half of the length of the primary route, 


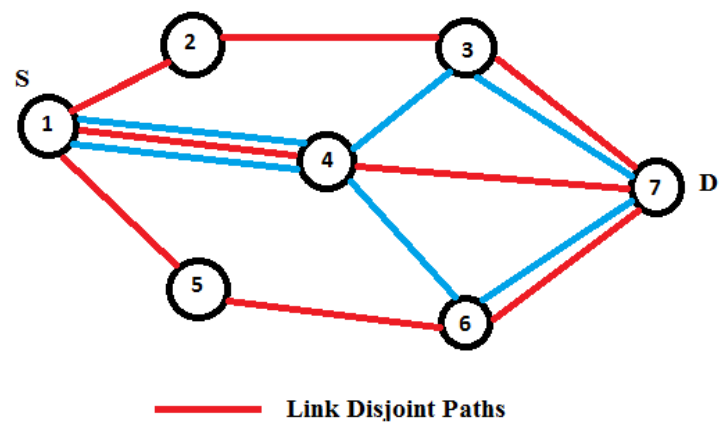

Figure 1. Link and Node Disjoint paths.

the disjointness condition is enforced and the packet is dropped if the partially discovered route does not comply. Also, the RReq backup Time to Live (TTL) is only one greater than the primary route length, again to reduce the extent to which the network is flooded [12].

\subsection{Node Disjoint Paths}

In node-disjoint paths no node is common other than the source and destination. This work enables discovery of two node disjoint paths from source to destination. In this protocol Hello messages have been used to identify two node disjoint paths. Three control packets are required for setup two node-disjoint routes, which reduce control overhead in the network and also setup backup route faster that reduces the end to end delay.

In QOSAR protocol, each node periodically broadcasts the HELLO messages to inform its neighbors that it has not moved away. When a node receives a HELLO message from a neighbor, a route to this neighbor is only added to the routing table when the neighbor does not already exists. If the neighbor exists, its lifetime is increased. When the network topology changes and HELLO, message does not received for a defined period of time the route expires.

In the above Figure 1, Intermediate node 4 is a primary route (1-4-7) from source to destination. If any failure occurred in the primary route it will take alternate route as (1-2-3-4) and (1-5-6-7) using node disjoint paths. If the primary and backup routes are selected in such a way that, they are sufficiently node disjoint then the route failure is minimized.

\section{Results and Discussion}

Simulations have been conducted in the environment of Network Simulator (NS2). The simulations start with the small network of 7 nodes.

Figure 2 shows small network with minimum number of nodes and the simulation parameters are listed in Table 1. We considering node 0 as a TCP traffic source and node 3 assumed to be TCP sink. This simulation results are presented in Table 2.

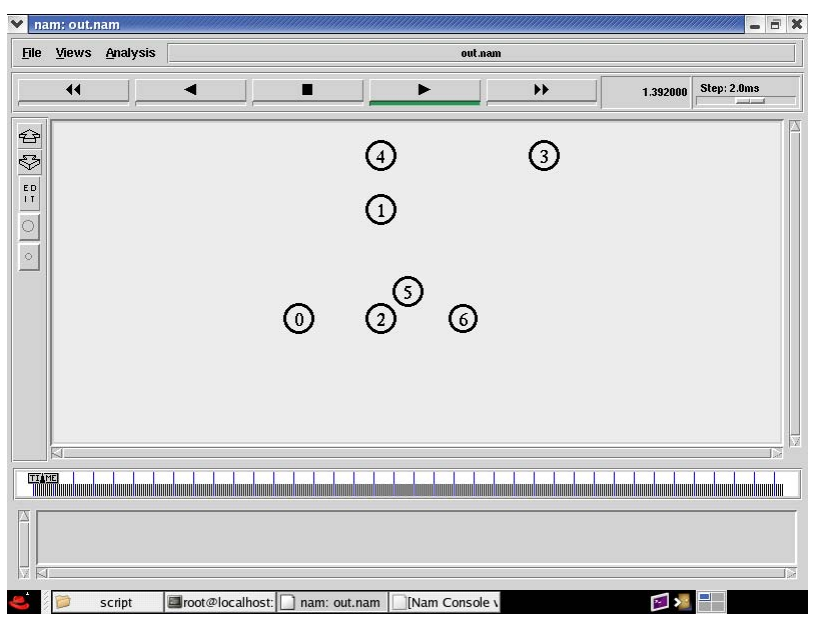

Figure 2. Small network with 7 nodes.

Table 1. Simulation Parameters Used for small network.

\begin{tabular}{cc}
\hline Parameter & Value \\
\hline Simulation area size & $100 \mathrm{~m} \times 100 \mathrm{~m}$ \\
Number of nodes & 7 \\
Number of traffic source & 1 \\
Simulation time & $100 \mathrm{~s}$ \\
Traffic source type & TCP \\
Propagation model & Shadowing \\
Path loss exponent & $2,2.2$ \\
\hline
\end{tabular}

Table 2. Simulation Results for small network.

\begin{tabular}{cccc}
\hline Path Loss & Parameter & AODV & Proposed Protocol \\
\hline \multirow{2}{*}{2} & Packets sent & 35983 & 42896 \\
& Packets received & 32547 & 42567 \\
& PDR & 90.451 & 99.233 \\
& Throughput & $4.72127 \times 10^{7}$ & $7.3808 \times 10^{7}$ \\
& Packets sent & 5132 & 19948 \\
& Packets received & 4196 & 18498 \\
& PDR & 81.7615 & 92.7311 \\
& Throughput & 876858 & $1.4964 \times 10^{7}$ \\
\hline
\end{tabular}

After observing the performance of QoSAR protocol in small network, then our simulations focused to observe the performance in large network. We have taken a network of 50 nodes in which node movements are random. Initially the network is as shown in Figure 3. The simulation parameters are presented in Table 3 . Three CBR flows with packet size of 1000 bytes are established between Nodes 37 and 18, Nodes 19 and 47 and Nodes 29 and 7. Propagation model used as shadowing model with path loss exponent varied from 1.6 to 2.8. The node movements are random and the speed of mobile node varied from 4 - $20 \mathrm{~m} / \mathrm{s}$.

Performances are analyzed for these two cases: 


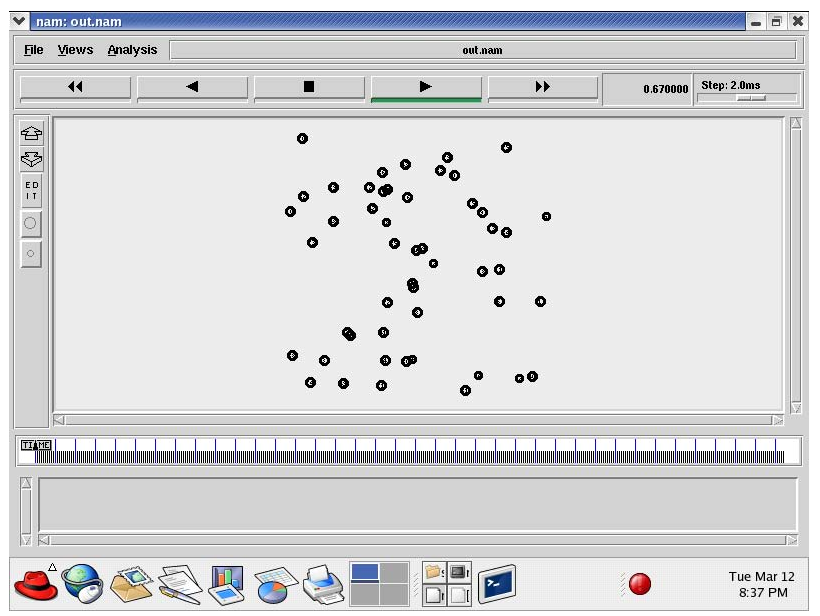

Figure 3. Large network with 50 nodes.

Table 3. Simulation Parameters Used for large network.

\begin{tabular}{cc}
\hline Parameter & Value \\
\hline Simulation area size & $500 \mathrm{~m} \times 500 \mathrm{~m}$ \\
Number of nodes & 50 \\
Node movement & Random \\
Node Speed when mobile & $4-20 \mathrm{~m} / \mathrm{s}$ \\
Node pause time when mobile & $50 \mathrm{~s}$ \\
Number of traffic source & 3 \\
Simulation time & $200 \mathrm{~s}$ \\
Traffic source type & CBR \\
Propagation model & Shadowing \\
Path loss exponent & 1.6 to 2.8 \\
\hline
\end{tabular}

- Finding backup routes by using Node Disjoint Paths termed in this paper as QoSAR Node Disjoint.

- Finding backup routes by using Link Disjoint Paths termed in this paper as QoSAR Link Disjoint.

The following graphs show the performance of QoSAR protocol.

The Figure 4 is clearly shows that the Packet Delivery Ratio (PDR) higher for proposed protocol (QOSAR) compared to AODV protocol. AODV protocol achieved low PDR due to link failure. This link failure can be achieved in our proposed protocol by the use of backup routes.

From Figure 5, the throughput assurance reliability achieved for proposed protocol increases with the speed of mobile nodes. It has been observed that QoSAR Node Disjoint and Link Disjoint protocol exhibits higher throughput than AODV protocol.

Still considering the Figure 6 QoSAR not only improves PDR and Throughput but it also reduces the delay considerably. From Figure 7, we can decide the Loss ratio also increases with increasing speed of mobile nodes in AODV protocol. Our proposed protocol gives much better performance compared to AODV.

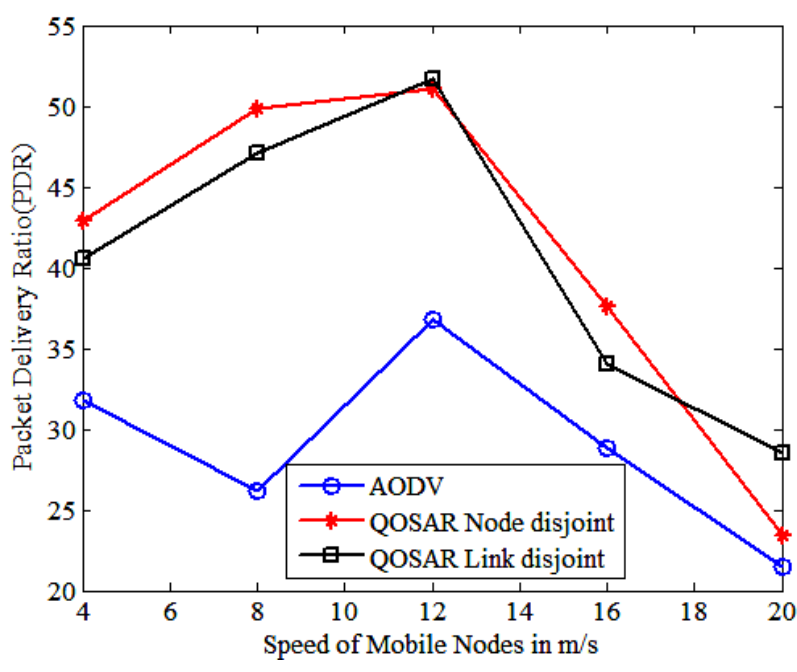

Figure 4. Speed vs Packet Delivery Ratio.

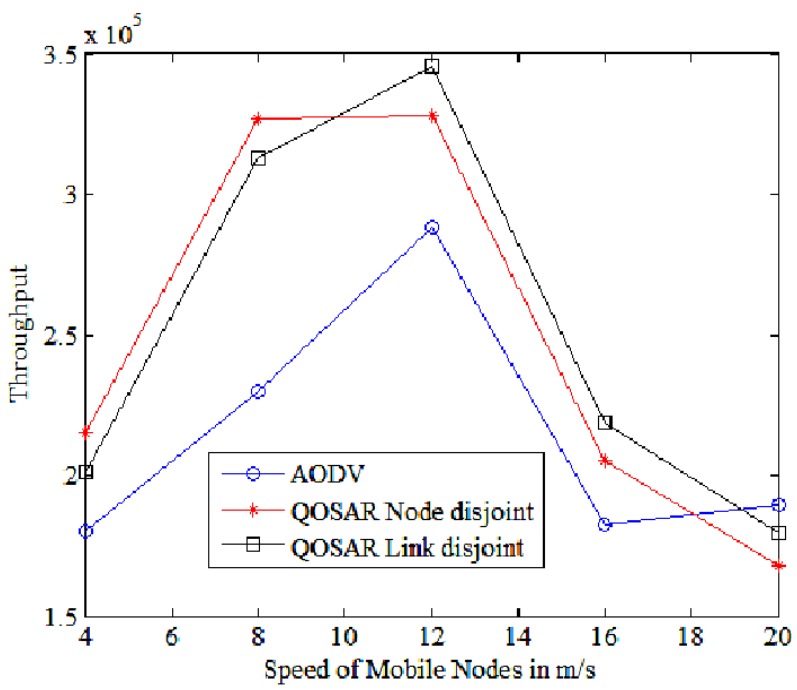

Figure 5. Speed vs Throughput.

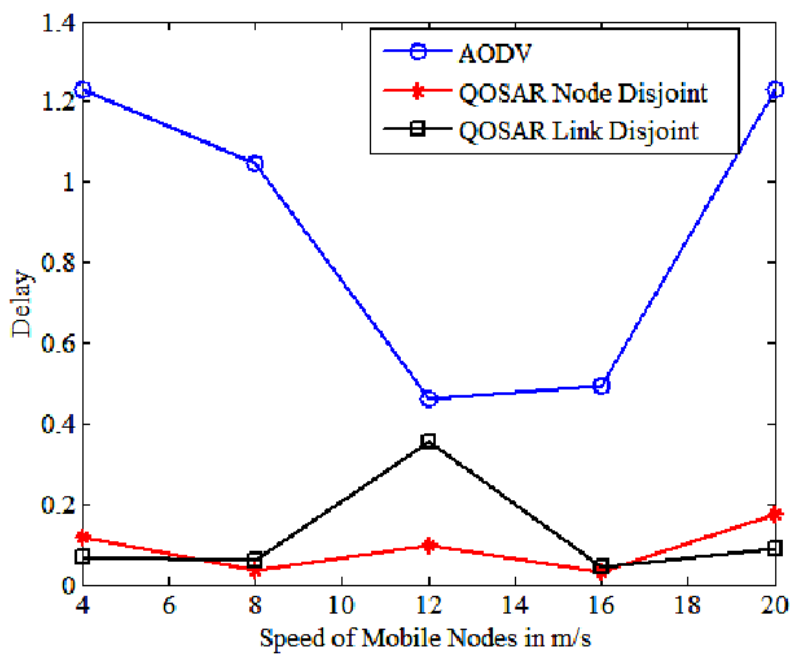

Figure 6. Speed vs Delay. 


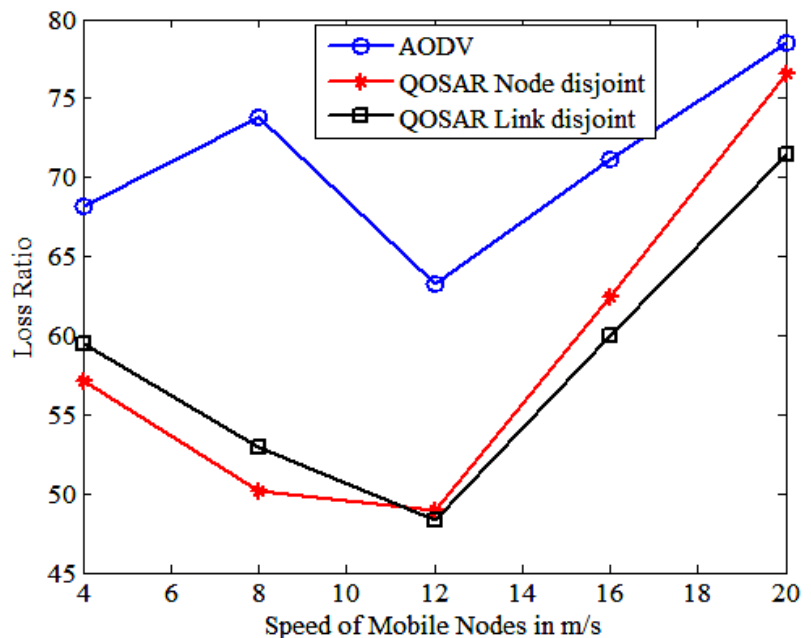

Figure 7. Speed vs Loss Ratio.

Figures 5, 6 and 8 confirm that the protocol proposed in this paper, in general, able to uphold throughput guarantees more in shadow fading environments. We can overcome route failure using backup routes. Comparisons show that QoSAR protocol provides better quality of service.

In this scenario we consider constant number of mobile nodes only variation in path loss exponential and the results obtained for packet delivery ratio. The Figure 8 shows that effects on the PDR and path loss exponential.

As shown in Figure 8. When increasing the path loss exponent the packet delivery ratio deceased to zero by both proposed and AODV protocol. Considering another case we can obtain high the packet delivery ratio for QoSAR protocol.

The simulation parameters for variable nodes shown in Table 4 and simulation results for variable nodes are shown in Table 5. In these simulation there are 10 - 50 mobile hosts randomly distributed in $100 \times 100$ meters. As per the performance analysis when increasing the number of mobile nodes in shadow fading environment the QOSAR disjoint paths achieve better performance compared to AODV. (i.e) considering the number of mobile node as 20 the PDR is achieved 95.2165 for AODV protocol and 99.8169 is achieved for QOSAR disjoint paths. While increasing the mobile nodes the source and destination has multiple paths to transmit and receive packets. So we can achieve higher throughput for QOSAR disjoints paths.

\section{Conclusion}

In MANET, it is difficult to provide QoS assurances due to node mobility, contention for channel access, lack of centralized coordination etc, as discussed previously. Simulation shows that backup routes improves Throughput and Packet Delivery Ratio (PDR) and also reduces Delay

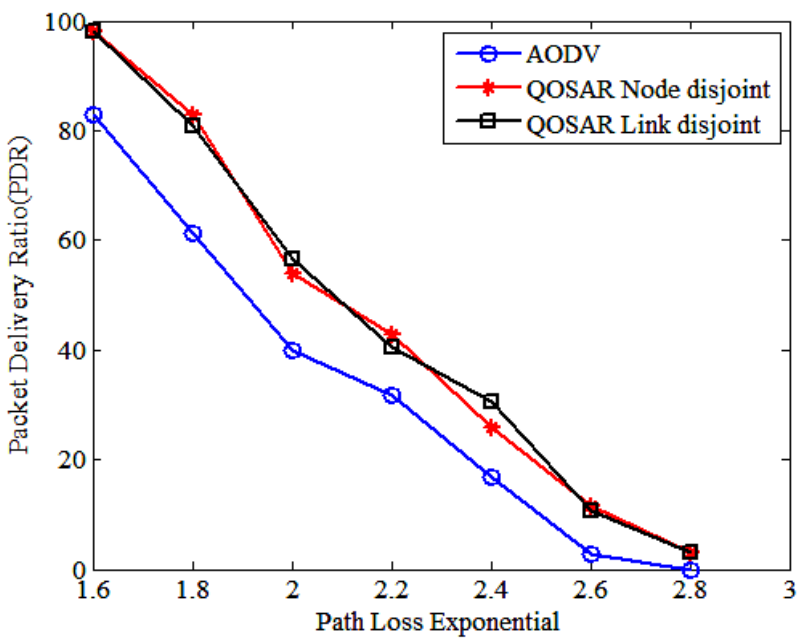

Figure 8. Path loss exponent vs Packet Delivery Ratio (PDR).

Table 4. Simulation Parameters for variable nodes.

\begin{tabular}{cc}
\hline Parameter & Value \\
\hline Simulation area size & $100 \mathrm{~m} \times 100 \mathrm{~m}$ \\
Number of nodes & $10-50$ \\
Number of traffic source & 1 \\
Simulation time & $100 \mathrm{~s}$ \\
Traffic source type & TCP \\
Propagation model & Shadowing \\
Path loss exponent & 2 \\
\hline
\end{tabular}

Table 5. Simulation Results for variable nodes.

\begin{tabular}{|c|c|c|c|}
\hline $\begin{array}{c}\text { Number of } \\
\text { nodes }\end{array}$ & Parameter & AODV & $\begin{array}{l}\text { QoSAR (Node } \\
\text { disjoint Paths) }\end{array}$ \\
\hline \multirow{4}{*}{10} & Packets sent & 36,089 & 54,879 \\
\hline & Packets received & 32,829 & 54,609 \\
\hline & PDR & 90.9668 & 99.508 \\
\hline & Throughput & $4.78226 \times 10^{7}$ & $1.21047 \times 10^{8}$ \\
\hline \multirow{4}{*}{20} & Packets sent & 51,448 & 66,644 \\
\hline & Packets received & 48,987 & 66,522 \\
\hline & PDR & 95.2165 & 99.8169 \\
\hline & Throughput & $1.01773 \times 10^{8}$ & $1.79061 \times 10^{8}$ \\
\hline \multirow{4}{*}{30} & Packets sent & 50,831 & 64,862 \\
\hline & Packets received & 48,630 & 64,740 \\
\hline & PDR & 95.67 & 99.8119 \\
\hline & Throughput & $1.002 \times 10^{8}$ & $1.69629 \times 10^{8}$ \\
\hline \multirow{4}{*}{40} & Packets sent & 54,190 & 66,269 \\
\hline & Packets received & 51,617 & 66,158 \\
\hline & PDR & 95.2519 & 99.8325 \\
\hline & Throughput & $1.13166 \times 10^{8}$ & $1.77077 \times 10^{8}$ \\
\hline \multirow{4}{*}{50} & Packets sent & 53,503 & 61,604 \\
\hline & Packets received & 51,083 & 61,512 \\
\hline & PDR & 95.4769 & 99.8507 \\
\hline & Throughput & $1.10681 \times 10^{8}$ & $1.53093 \times 10^{8}$ \\
\hline
\end{tabular}


and loss ratio in shadow fading environments. As per the performance analysis the QoSAR protocol gives better performance in shadow fading environments.

However, it was found that with severe shadowing induced signal strength fluctuations, the backup routes was less significant, although merely proactively seeking backup routes still improved to achieve QoS. This suggests that routing protocols benefit from proactively requiring that backup routes exist. However, the more than one backup route is counter-productive due to the excess overhead incurred when initiating state information setup. The required level of link disjointness between data sessions' primary and backup routes was also studied [12]. Thus it is suggested that by using node disjoint and link disjoint paths, we can achieve better Quality of Service (QoS) in shadow fading environments.

\section{REFERENCES}

[1] R. Ramanathan, J. Redi and B. Technologies, “A Brief Overview of Ad-hoc Networks: Challenges and Directions,” IEEE Communications Magazine, Vol. 40, No. 5, 2002, pp. 20-22.

http://dx.doi.org/10.1109/MCOM.2002.1006968

[2] R. Shi and Y. Deng, "An Improved Scheme for Reducing the Latency of AODV in Mobile Ad Hoc Networks," 9th International Conference for Young Computer Scientists, IEEE Computer Society, 2008, pp. 594-598.

[3] H.-W. Tsai, T.-S. Chen and C.-P. Chu, “An On-Demand Routing Protocol with Backtracking for Mobile Ad Hoc Networks," WCNC IEEE Communication Society, 2004, pp. 1557-1561.

[4] G. Di Caro, F. Ducatelle and L. M. Gambardella, "Special Issue on Self-Organization in Mobile Networking AntHocNet: An Adaptive Nature-Inspired Algorithm for Routing in Mobile Ad Hoc Networks”, European Transactions on Telecommunications, Vol. 16, 2005, pp. 443455. http://dx.doi.org/10.1002/ett.1062

[5] R. Bai and M. Singhal "DOA: DSR over AODV Routing for Mobile Ad Hoc Networks," IEEE Transaction on Mobile Computing, Vol. 5, No. 10, 2006, pp. 1403-1416.

[6] L. Hanzo II. and R. Tafazoli, "A Survey of QoS Routing Solutions for MANETs,” IEEE Communications Surveys and Tutorials, Vol. 9, No. 2, 2007, pp. 50-70. http://dx.doi.org/10.1109/COMST.2007.382407

[7] L. Hanzo II. and R. Tafazolli, “Admission Control Schemes for 802.11-Based Multi-Hop Mobile Ad-hoc Networks: A Survey," IEEE Communications Surveys \& Tutorials, Vol. 11, No. 4, 2009.

[8] C. E. Perkins and E. M. Royer, "Ad Hoc On-Demand Distance Vector Routing (AODV),” IETF RFC 3561, 2003.

[9] C. E. Perkings, E. M. Royer and S. R. Das, "Performance Comparison of Two On-Demand Routing Protocols for Ad Hoc Networks," IEEE Personal Communications, Febuary 2001.

[10] L. Chen and W. B. Heinzelman, "QoS-Aware Routing Based on Bandwidth Estimation for Mobile Ad Hoc Networks" IEEE Journal on Selected Areas in Communications, Vol. 23, No. 3, March 2005.

[11] D. B. Johnson and D. A. Maltz, "Dynamic Source Routing in Ad Hoc Wireless Networks,” Mobile Computing, T. Imielinski and H. Korth, Eds., Kluwer Academic, Vol. 353, 1996, pp. 153-181.

[12] L. Hanzo and R. Tafazolli, "QoS-Aware Routing and Admission Control in Shadow-Fading Environments for Multirate Manets," IEEE Transactions on Mobile Computing, Vol. 10, No. 5, 2011. http://dx.doi.org/10.1109/TMC.2010.208

[13] L. Hanzo and R. Tafazolli, "A Survey of QoS Routing solutions for Mobile Adhoc Networks," IEEE Communications Surveys and Tutorials, Vol. 9, No. 2, 2007, pp. 50-70.

http://dx.doi.org/10.1109/COMST.2007.382407 